\title{
Alterstice
}

Revue internationale de la recherche interculturelle

International Journal of Intercultural Research

Revista International de la Investigacion Intercultural

\section{Pour la réussite des jumelages interculturels : leadership pédagogique et institutionnel}

\section{Marie-Cécile Guillot et Nicole Carignan}

Volume 8, numéro 1, 2018

Jumelage interculturel et pédagogie universitaire

URI : https://id.erudit.org/iderudit/1052605ar

DOI : https://doi.org/10.7202/1052605ar

Aller au sommaire du numéro

Éditeur(s)

Alterstice

ISSN

1923-919X (numérique)

Découvrir la revue

Citer cet article

Guillot, M.-C. \& Carignan, N. (2018). Pour la réussite des jumelages

interculturels : leadership pédagogique et institutionnel. Alterstice, 8(1), 25-36. https://doi.org/10.7202/1052605ar

\section{Résumé de l'article}

Bien que l'intégration des immigrants soit une priorité au Québec, de nombreux immigrants ont malheureusement peu de contact avec la communauté francophone majoritaire. Ce manque de réseaux francophones constitue un frein important à leur apprentissage et à leur usage de la langue française. Devant cette situation, l'Université du Québec à Montréal (UQAM) a décidé de mettre en place les jumelages interculturels, qui sont des activités d'échange et d'apprentissage réunissant des personnes immigrantes apprenant le français (dans des cours de communication orale et écrite, de phonétique et de grammaire) et des francophones étudiant en éducation, en psychologie, en travail social et en communication. Il s'agit d'une occasion pour les immigrants non francophones de pratiquer la langue et, pour les membres de la société d'accueil, d'être en contact avec des immigrants afin de faciliter leur intégration. Cet article présente le contexte du Québec, les objectifs, les caractéristiques, l'historique et la pertinence des jumelages interculturels, les caractéristiques des participants au jumelage, les assises conceptuelles et théoriques permettant de comprendre les relations intergroupes pour une société qui se veut inclusive, le soutien institutionnel et la mobilisation des gestionnaires pour le leadership partagé, l'engagement des enseignants, les types de jumelage et les retombées. Ces jumelages interculturels ont contribué à faire fondre le mur d'incompréhension, à débusquer les préjugés, à reconnaître la diversité ethnoculturelle, à promouvoir l'enrichissement culturel réciproque autant des immigrants qui apprennent le français que des francophones. Les jumelages favorisent la cohésion sociale et le vivre-ensemble.
Ce document est protégé par la loi sur le droit d'auteur. L’utilisation des services d’Érudit (y compris la reproduction) est assujettie à sa politique d'utilisation que vous pouvez consulter en ligne.

https://apropos.erudit.org/fr/usagers/politique-dutilisation/ 


\title{
7
}

ARTICLE THÉMATIQUE

\section{Pour la réussite des jumelages interculturels : leadership pédagogique et institutionnel}

\author{
Marie-Cécile Guillot ${ }^{1}$ et Nicole Carignan ${ }^{1}$
}

\section{Résumé}

Bien que l'intégration des immigrants soit une priorité au Québec, de nombreux immigrants ont malheureusement peu de contact avec la communauté francophone majoritaire. Ce manque de réseaux francophones constitue un frein important à leur apprentissage et à leur usage de la langue française. Devant cette situation, l'Université du Québec à Montréal (UQAM) a décidé de mettre en place les jumelages interculturels, qui sont des activités d'échange et d'apprentissage réunissant des personnes immigrantes apprenant le français (dans des cours de communication orale et écrite, de phonétique et de grammaire) et des francophones étudiant en éducation, en psychologie, en travail social et en communication. II s'agit d'une occasion pour les immigrants non francophones de pratiquer la langue et, pour les membres de la société d'accueil, d'être en contact avec des immigrants afin de faciliter leur intégration. Cet article présente le contexte du Québec, les objectifs, les caractéristiques, l’historique et la pertinence des jumelages interculturels, les caractéristiques des participants au jumelage, les assises conceptuelles et théoriques permettant de comprendre les relations intergroupes pour une société qui se veut inclusive, le soutien institutionnel et la mobilisation des gestionnaires pour le leadership partagé, l'engagement des enseignants, les types de jumelage et les retombées. Ces jumelages interculturels ont contribué à faire fondre le mur d'incompréhension, à débusquer les préjugés, à reconnaître la diversité ethnoculturelle, à promouvoir l'enrichissement culturel réciproque autant des immigrants qui apprennent le français que des francophones. Les jumelages favorisent la cohésion sociale et le vivreensemble.

\section{Rattachement des auteures \\ ${ }^{1}$ Université du Québec à Montréal, Montréal, Canada}

\section{Correspondance}

guillot.marie-cecile@uqam.ca

\section{Mots clés}

jumelage interculturel, apprentissage et enseignement du français, éducation interculturelle, relations intergroupes, diversité ethnoculturelle, immigration, intégration, leadership pédagogique et institutionnel

\section{Pour citer cet article}

Guillot, M.-C. et Carignan, N. (2018). Pour la réussite des jumelages interculturels : leadership pédagogique et institutionnel. Alterstice, 8(1), 25-36. 


\section{Introduction}

On ne peut comprendre la portée des jumelages interculturels sans comprendre la place de l'immigration dans la société québécoise. Quelle est la responsabilité des membres de la société d'accueil ? Quelle est la responsabilité des immigrants ? Quels sont leurs défis respectifs ? De quelle façon les milieux d'enseignement peuvent-ils jouer leur rôle relativement à l'intégration des immigrants? Dans un tel contexte, quelle est la contribution des jumelages interculturels ? C'est à ces questions que ce texte tente de répondre, en présentant le contexte du Québec, les objectifs, les caractéristiques, l'historique et la pertinence des jumelages interculturels, les participants, les assises conceptuelles et théoriques, le soutien institutionnel, l'engagement des enseignants, les types de jumelage et les retombées.

\section{Le contexte du Québec}

Dans le rapport du Conseil supérieur de la langue française (CSLF) rédigé par St-Laurent et El-Geledi en 2011, il est stipulé que, dans le contexte du Québec, le français est la langue officielle et la langue du groupe linguistique majoritaire. Conséquemment, l'apprentissage du français et l'intégration linguistique des nouveaux arrivants non francophones représentent tout un défi. Il y est mentionné que le "Québec a besoin de l'immigration pour son développement actuel et futur » (p. 5), d’où l'importance de compter sur l'immigration pour sa pérennité.

Or ce rapport a révélé deux aspects qui méritent toute notre attention, soit la nécessité de sensibiliser les enseignants de français à la réalité que vivent les immigrants et de valoriser l'intégration des immigrants dans des réseaux francophones. Cette étude exploratoire permet de décrire les perceptions de 11 intervenants travaillant dans des organismes de services d'accueil et d'aide aux immigrants et 22 immigrants relativement à la langue française, à leur parcours migratoire, à la façon dont ils se sont intégrés au Québec et à leurs difficultés liées à leur absence de connaissance ou leur manque de maîtrise du français. Bien que les résultats de cette étude ne puissent être généralisés compte tenu de la taille de l'échantillon, ils révèlent des aspects de l'accueil des immigrants qui méritent réflexion.

Selon St-Laurent et El-Geledi (2011), la sensibilisation des enseignants de français à la réalité que vivent les immigrants a montré du doigt " l'attitude parfois trop "pro-francophone" (ou "anti-anglophone") des professeurs " (p. 75). Une répondante qui a participé à l'étude a souligné qu'il fallait « dépolitiser » l'enseignement du français et développer de nouvelles pratiques d'enseignement.

Ce [les enseignants] sont des fervents défenseurs de la langue française. [...] Je veux bien défendre la langue française, mais il faut du respect pour la personne qui est devant soi et qui essaie de l'apprendre. Il faut la comprendre un peu. Parce que, souvent, l'enseignant a un discours qui est politique. La personne [immigrante] ne le comprend pas, elle est juste blessée (StLaurent et El-Geledi, 2011, p. 75).

Les enseignants de français sont confrontés à la réalité socioculturelle vécue par les immigrants et au rejet qu'ils ressentent. Une meilleure connaissance des défis que doivent relever les immigrants peut permettre aux enseignants d'adapter leurs stratégies pédagogiques et de développer de nouvelles pratiques :

Pour être franc, j'aimais plus [la langue française] avant de venir ici [...] En réalité, je l'aimais plus que ma [propre] langue. [...] Mais quand on arrive ici, tout change à cause de l'attitude [des francophones]. (St-Laurent et El-Geledi, 2011, p. 62)

De plus, selon ce rapport, l'intégration des immigrants dans des réseaux francophones doit devenir une priorité. À titre d'exemple, une autre répondante a révélé que

[s] l'apprentissage du français représente un grand défi pour les immigrants, la pratique de la langue et la possibilité d'entrer dans des réseaux francophones le sont tout autant. C'est pourquoi les immigrants proposent de promouvoir les activités de rapprochement culturel et de jumelage pour leur permettre de rencontrer d'autres francophones et de pratiquer leur français tout en créant des liens avec eux (St-Laurent et El-Geledi, 2011, p. 75).

Les immigrants de l'étude déplorent de ne pas connaître de francophones, de ne pas pouvoir consolider leurs connaissances en français, ni de percevoir l'utilité de la langue puisqu'ils ne l'utilisent pas. Ils déplorent également 
l'attitude de nombreux francophones à leur égard qui " "s'adaptent" à eux en optant spontanément pour l'anglais ou, au contraire, leur reprochent leur faible maîtrise du français " (St-Laurent et El-Geledi, 2011, p. 67). Malheureusement, de nombreux immigrants sont peu ou ne sont pas intégrés à la communauté francophone et cette situation constitue un frein important à leur apprentissage et à leur usage de la langue française.

Une intervenante travaillant dans un organisme offrant des services d'aide à l'emploi rapporte :

Ils trouvent ça difficile de [nouer] des amitiés avec des Québécois. J'entends ça souvent. J'ai eu un client du Mexique qui parle quand même bien le français même s'il est plus anglophone. II m'a dit: "Je ne réussis pas. Je pense qu'ils ne veulent pas de moi. Au travail non plus, je n'y arrive pas. J'ai juste des amis immigrants". Ce n'est pas la première fois que j'entends ça. Je ne sais pas si c'est les [Québécois] qui ne s'intéressent pas [aux immigrants] ou les immigrants qui ne vont pas vers les Québécois [...]. C'est plus les Québécois [francophones qui sont en cause]. (St-Laurent et El-Geledi, 2011, p. 62)

\section{Les objectifs des jumelages interculturels}

C'est dans le but de sensibiliser les enseignants à la réalité vécue par les immigrants et de valoriser l'intégration des immigrants dans des réseaux francophones que nous avons mis en place les jumelages interculturels. Ces rencontres nous ont conduits à mieux comprendre les relations intergroupes, à débusquer les préjugés et la discrimination, à proposer un rapport à la langue française positif, à favoriser des contacts entre Québécoises et Québécois de toutes origines, et aussi à développer de nouvelles pratiques d'enseignement et d'apprentissage.

\section{Les jumelages interculturels}

Mais avant toute chose, que sont les jumelages interculturels à l'Université du Québec à Montréal (UQAM) ? II s'agit d'activités d'échange et d'apprentissage réunissant des personnes immigrantes apprenant le français (dans des cours de communication orale et écrite, de phonétique et de grammaire) et des francophones étudiant, entre autres, en éducation, en psychologie, en travail social et en communication. Chez les immigrants adultes qui apprennent le français à l'université, ces jumelages visent aussi leur intégration à leur société d'accueil. Chez les futurs enseignants du primaire, du secondaire et du collégial, les carriérologues, les psychologues, les travailleurs sociaux et les professionnels en communication, les jumelages visent à les sensibiliser à la diversité ethnoculturelle et à les outiller pour travailler avec des personnes d'autres cultures.

Depuis plus d'une décennie, les jumelages interculturels ont contribué à faire fondre le mur d'incompréhension, à débusquer les préjugés, à reconnaître la diversité ethnoculturelle et à contribuer à l'enrichissement culturel réciproque autant des immigrants qui apprennent le français que des francophones.

\section{L'historique des jumelages interculturels}

Le premier jumelage, qui a eu lieu en 2002 entre des étudiants de l'École de langues et des étudiants en éducation, est né d'un constat. La directrice de l'École de langues réalise que, malgré une formation soutenue, ses étudiants rencontrent très peu d'étudiants francophones de la majorité d'accueil sur le campus tandis que sa collègue de la Faculté des sciences de l'Éducation réalise que la majorité de ses étudiants ont grandi et continuent d'évoluer dans un milieu unilingue et monoculturel francophone où la présence des immigrants est rare. Cette réalité a donné naissance à l'idée que les étudiants devaient se rencontrer afin d'apprendre à se connaître.

Depuis, c'est plus de 15000 jumelles et jumeaux qui ont participé à des jumelages interculturels à l'UQAM. En plus de mobiliser les enseignants du certificat en français langue seconde (FLS) de l'École de langues et ceux de différents départements (éducation et formation spécialisées, carriérologie, didactique des langues, travail social, psychologie et communication), ces jumelages permettent de mobiliser les étudiants admis dans ces programmes. Au total, une cinquantaine de professeurs, maîtres de langue ou personnes chargées de cours ont pu offrir cette expérience hors du commun à leurs étudiants.

Au fil du temps, ces jumelages ont permis autant aux étudiants qu'aux enseignants d'apprendre ensemble et de découvrir la richesse des uns et des autres. 


\section{La pertinence des jumelages interculturels}

Les immigrants qui arrivent au Québec ont l'obligation d'apprendre le français afin de trouver un emploi, de refaire des études, de se conformer aux exigences des ordres professionnels ou d'être en mesure d'encadrer leurs enfants d'âge scolaire. C'est ainsi que beaucoup apprennent le français, dans un premier temps, dans des cours de francisation donnés soit par le ministère de I'Immigration, de la Diversité et de I'Inclusion (MIDI), soit par des commissions scolaires, puis, par la suite, à l'université. Outre l'aspect interculturel de l'activité, il importe de mentionner qu'elle constitue une activité concrète en termes d'enseignement/apprentissage d'une langue seconde. En effet, comme le souligne Bourguignon (2009), « Apprendre les langues dans une perspective actionnelle signifie que c'est à travers l'usage que se fait l'apprentissage " (p. 59). Elle propose aussi un " scénario d'apprentissageaction [qui comprend] cinq activités de communication langagière [...] : lire et écouter, interaction orale ou interaction écrite, parler et écrire) » (p. 72). Le jumelage interculturel s'inscrit dans cette démarche puisqu'il fait appel à ces cinq activités. Enfin, il est important de rappeler que « l'on n'apprend pas une langue (juste) pour le plaisir d'apprendre une langue, mais en vue de s'intégrer dans une communauté différente et pour y devenir, autant que faire se peut, un acteur social à part entière. » (Rosen, 2007, p. 17-18).

\section{Les participants aux jumelages interculturels}

Au Québec, les écrits scientifiques et gouvernementaux reconnaissent trois groupes : les francophones, les anglophones et les allophones. Les allophones, qui sont des personnes dont la langue maternelle n'est ni le français ni l'anglais, peuvent être des citoyens canadiens issus de l'immigration ou des immigrants reçus en attente de l'obtention de leur citoyenneté. Ils peuvent être bilingues francophones ayant appris le français comme langue seconde (FL2), ou encore bilingues anglophones, ayant appris l'anglais comme langue seconde (EL2). Ils peuvent aussi être trilingues ayant acquis la connaissance du français et de l'anglais (MIDI, 2014). Les anglophones sont ceux qui ont l'anglais comme langue maternelle ou comme langue d'usage.

Dans le contexte canadien et québécois, est francophone toute personne dont la langue maternelle et la langue d'usage sont le français. Les francophones désignent ici, de plus, les étudiants qui participent aux jumelages et qui ne sont pas étudiants en français langue seconde (FLS). En réalité, certains sont allophones et ont déjà vécu le processus d'immigration. Le terme non-francophone, utilisé sans discrimination, désigne ici les étudiants inscrits dans le programme de FLS à l'École de langues de I'UQAM.

Par ailleurs, les francophiles et les francotropes font aussi partie de la majorité d'accueil au Québec. Une personne francophile manifeste un grand intérêt par rapport à la langue française et la culture qu'elle véhicule. Ainsi, Bélanger, Sabourin et Lachapelle (2011) ont défini comme francotropes,

les allophones nés dans un pays membre de l'Organisation internationale de la francophonie (OIF, essentiellement des anciennes colonies françaises ou belges), ou dont la langue maternelle est une langue romane ou une langue parlée dans un des pays de l'OIF. Puisque l'orientation linguistique est définie par la langue maternelle et le pays de naissance des parents, l'opposition francotrope/non-francotrope est antérieure à l'immigration. (p. 121-122)

En fait, le Québec, qui est responsable de la sélection des immigrants qui s'établissent sur son territoire, souhaite attirer et retenir des immigrants qui pourront plus facilement apprendre le français et s'intégrer à la majorité francophone. Dans le cadre des jumelages interculturels, certains des étudiants faisant partie du groupe des francophones sont ainsi des allophones francophiles ou francotropes. Les personnes francophiles manifestent un grand intérêt pour la langue française et les personnes francotropes sont nées dans un pays membre de l'Organisation internationale de la francophonie ou dont la langue maternelle est une langue romane. En fait, les jumelages interculturels répondent aux besoins des « accueillis » comme à ceux des « accueillants ».

\section{Les assises conceptuelles et théoriques des jumelages interculturels}

Les jumelages interculturels reposent d'abord sur les concepts d'endogroupe, d'exogroupe, de préjugé, de discrimination et de stigmatisation. L'endogroupe est un groupe composé d'individus qui se catégorisent comme membres du groupe d'appartenance auquel ils s'identifient. L'exogroupe est un groupe composé d'individus qui se catégorisent comme membres d'un groupe d'appartenance différent du leur et auquel ils ne s'identifient pas (Bourhis 
et Leyens, 1999). C'est dans cette perspective que ces jumelages favorisent la rencontre des francophones majoritaires dans la société d'accueil et de centaines d'immigrants venus à l'université pour apprendre le français. Dans l'optique des francophones, être du côté des " accueillants " signifie partager les caractéristiques d'un endogroupe considéré comme " notre " groupe, c'est-à-dire le " Nous ». Face aux " accueillants " se retrouvent les " accueillis », qui font partie d'un exogroupe différent de celui des francophones majoritaires.

Mieux comprendre les relations entre les membres de l'endogroupe et ceux de l'exogroupe permet de saisir ce qu'est le préjugé et les types de discrimination qui gangrènent les sociétés contemporaines, en particulier le racisme, la xénophobie, le linguicisme, l'antisémitisme, l'arabophobie, le sexisme, l'homophobie, l'âgisme (Bourhis et Gagnon, 2006). Le préjugé est un jugement a priori, un parti pris, une opinion préconçue visant l'ensemble des membres d'un exogroupe qui s'appuie sur une généralisation erronée et rigide (Allport, 1954). Ce jugement hâtif, qui ne permet pas de reconnaître les différences individuelles existant à l'intérieur d'un groupe (Brown, 2010), soutient une attitude négative ou une prédisposition à adopter un comportement négatif envers tous les membres d'un exogroupe dévalorisé.

Entretenir des préjugés à l'égard des membres d'un groupe dévalorisé favorise un comportement négatif qui conduit à faire de la discrimination (Bourhis et Gagnon, 2006). La discrimination institutionnelle peut être directe, indirecte ou systémique. La discrimination directe, qui est volontaire et évidente dans le fonctionnement d'une organisation, résulte du traitement inégal réservé à une personne ou à un groupe dévalorisé. Par exemple, on a tous entendu des histoires d'individus, issus de communautés juives, noires ou chinoises, interdits de travailler dans les banques. La discrimination indirecte, qui est involontaire et non intentionnelle, résulte d'une règle ou d'une loi qui se veut " neutre " puisqu'elle s'applique à tous de la même façon, et peut produire un effet discriminatoire. Par exemple, les corps policiers ont dû changer certains critères d'éligibilité (taille et poids) pour permettre l'accès des femmes dans la profession. La discrimination systémique, qui est issue de conséquences involontaires de systèmes et de pratiques d'emplois établis comme des tests d'aptitudes culturellement biaisés, a pour effet de gêner l'accès de certaines catégories d'emplois aux membres de minorités culturelles. Prenant acte des effets néfastes de la discrimination institutionnelle, la plupart des États de droit ont adopté des chartes de droits et libertés.

En plus de la discrimination institutionnelle, les études de Croizet et Leyens (2003) ont montré que la discrimination sociale se manifeste entre les individus dans l'ensemble des relations interpersonnelles. Dans l'espace public, des individus peuvent subir de la stigmatisation, qui est un comportement négatif injustifié envers une personne à cause de caractéristiques personnelles telles que son poids, sa taille, un physique ingrat, une déficience comportementale, physique ou intellectuelle. Ces études ont aussi démontré que les individus victimes de préjugés ou de discrimination souffraient d'une estime de soi négative, se sentaient plus tristes, stressés et dépressifs que ceux qui ne subissaient pas ces abus (Croizet et Leyens, 2003).

Par ailleurs, aux États-Unis et en Grande-Bretagne, plusieurs études épidémiologiques ont révélé que les victimes chroniques de préjugés ou de discrimination (par exemple, les Africains-Américains et les musulmans) développent des sentiments d'impuissance et de perte de contrôle dans leur vie quotidienne, ce qui les rend plus susceptibles de souffrir d'hypertension, de haut taux de cholestérol, de maladies cardiaques, de surpoids et d'obésité (James et Thomas, 2000; Johnson et Lordan, 2012).

De plus, plusieurs études ont permis de démontrer que la catégorisation «Eux-Nous » et l'identification à son propre groupe d'appartenance (endogroupe) suffisent pour déclencher les préjugés et les comportements discriminatoires (Tajfel et Turner, 1986).

Les jumelages interculturels tels qu'ils sont proposés à l'UQAM visent à réduire les préjugés que des étudiants peuvent entretenir les uns envers les autres et à les sensibiliser aux conséquences négatives sur la santé physique et psychologique de ceux qui subissent différents types de discrimination. Afin de réduire les préjugés et la discrimination, Pettigrew et Tropp (2011) ainsi que Paluck et Green (2009) inspirent notre démarche conceptuelle et théorique. Nos assises conceptuelles et théoriques s'appuient sur les trois conditions " gagnantes » de l'hypothèse du contact d'Allport (1954). Dans le contexte des jumelages, ces trois conditions sont : 1) les étudiants de FLS ont un statut équivalant à celui des étudiants francophones en éducation, 2) le but commun des étudiants jumelés est de 
communiquer en français et d'échanger sur leur culture respective et 3 ) les étudiants doivent décrire et analyser les échanges effectués lors du jumelage pour rédiger leur travail de réflexion obligatoire. Puisque la volonté individuelle ne suffit pas pour assurer la pleine réussite du contact entre les groupes, la troisième condition préconise un soutien institutionnel des activités de jumelage, c'est-à-dire que les étudiants y participant doivent répondre aux exigences de leur programme universitaire respectif (Allport, 1954).

De leur côté, les orientations d'acculturation (Bourhis, Moïse, Perreault et Senécal, 1997) permettent de mieux comprendre la dynamique relationnelle entre les étudiants francophones en éducation et en FLS durant les jumelages. Comme nous le verrons plus loin, une meilleure compréhension de cette dynamique relationnelle amène au développement des compétences de communication interculturelle (Lussier, 2005, 2011). En ce qui concerne le processus d'acculturation, il se déclenche lorsque deux groupes de cultures différentes se trouvent en contact soutenu et direct l'un avec l'autre. Ce contact entraîne des changements dans les modèles culturels initiaux des groupes en présence (Berry, 1997; Sam et Berry, 2006). Ces changements peuvent être psychologiques, culturels, linguistiques et religieux.

L'acculturation se produit rarement entre des groupes de pouvoir et de statuts égaux. Ces contacts intergroupes s'établissent le plus souvent entre le groupe majoritaire de la société d'accueil et les groupes minoritaires qui sont plus vulnérables et souvent issus de l'immigration (Bourhis et El-Geledi, 2010). Chaque orientation d'acculturation réfère à une combinaison d'attitudes, de croyances et d'intentions de comportement qui guident les façons de penser et d'agir des individus, autant ceux qui appartiennent à la majorité que ceux qui appartiennent à la ou aux minorités. Les orientations d'acculturation vont permettre de définir le type de relations que les membres des communautés d'accueil et les immigrants vont entretenir les uns envers les autres. Ces types de relations peuvent être harmonieuses, problématiques ou conflictuelles. En ce qui concerne les jumelages interculturels, I'UQAM veut favoriser des relations harmonieuses entre les étudiants francophones (majoritaires) et les étudiants immigrants (minoritaires) qui apprennent le français.

Le modèle d'orientations d'acculturation interactif (MAI) propose six attitudes et tendances comportementales que les membres de la communauté d'accueil peuvent adopter envers les immigrants :

- individualisme

- intégrationnisme

- intégrationnisme de transformation

- assimilationnisme

- ségrégationnisme

- exclusionnisme.

L'individualisme est l'orientation d'acculturation " adoptée par les membres de la communauté d'accueil lorsqu'ils se définissent et définissent les autres en tant qu'individus plutôt que comme membres de catégories sociales "EuxNous". Dans leurs relations avec les immigrants, ce sont les caractéristiques personnelles, les compétences et l'accomplissement individuel qui prévalent, et non le fait d'appartenir à un groupe culturel, linguistique ou religieux en particulier » (Bourhis, Carignan et Sioufi, 2015, p. 18).

L'intégrationnisme valorise « le maintien de la culture d'origine des immigrants tout en favorisant l'adoption de la culture d'accueil par les immigrants " (Bourhis, Carignan et Sioufi, 2015, p. 18). Pour sa part, l'intégrationnisme de transformation pratiquée par les membres de la communauté d'accueil est leur acceptation de " modifier certains aspects de leur culture et de leurs pratiques institutionnelles afin de faciliter l'intégration des immigrants " (Bourhis, Carignan et Sioufi, 2015, p. 18). L'endossement de l'individualisme, de l'intégrationnisme et de l'intégrationnisme de transformation favorise des relations harmonieuses avec les immigrants parce que ces trois orientations d'acculturation sont accueillantes envers eux.

Quant aux membres de la communauté d'accueil qui adhèrent à l'assimilationnisme, ils « s'attendent à ce que les immigrants renoncent à leur culture d'origine pour adopter endosser la culture de la majorité d'accueil » (Bourhis, Carignan et Sioufi, 2015, p. 18). Les assimilationnistes, qui n'acceptent pas que les immigrants conservent leur 
culture, " considèrent que les immigrants doivent s'assimiler linguistiquement, culturellement et religieusement à la société d'accueil » (Bourhis, Carignan et Sioufi, 2015, p.18).

Les tenants du ségrégationnisme, quant à eux, acceptent que les immigrants conservent leur héritage culturel. Toutefois, ils « ne souhaitent pas que les immigrants adoptent ou influencent la culture d'accueil " (Bourhis, Carignan et Sioufi, 2015, p.18) et tendent à éviter les relations avec eux, préférant qu'ils se regroupent dans leurs quartiers.

Enfin, ceux qui adhèrent à l'exclusionnisme "refusent que les immigrants préservent leur culture d'origine et s'opposent aussi à ce qu'ils adoptent ou influencent la culture d'accueil. Les exclusionnistes considèrent que les immigrants risquent de " contaminer » l'authenticité de la culture d'accueil » (Bourhis, Carignan et Sioufi, 2015, p.1819). L'endossement de ces trois orientations (assimilationniste, ségrégationniste et exclusionniste) entraîne des relations problématiques ou conflictuelles avec les immigrants parce qu'elles sont négatives envers eux et risquent d'avoir un impact sur les rencontres lors des jumelages.

Il a été démontré que les étudiants universitaires endossent surtout l'individualisme et l'intégrationnisme. Ceci pourrait s'expliquer par la culture organisationnelle universitaire qui valorise la méritocratie et l'accomplissement personnel indépendamment de l'origine ethnique, culturelle ou religieuse (Bourhis, Barrette, El-Geledi et Schmidt, 2009). On peut ainsi conclure que les étudiants individualistes et intégrationnistes sont moins ethnocentriques et plus favorables à intégrer les immigrants dans leurs réseaux. Pour eux, les relations avec les immigrants sont positives et harmonieuses. Par contre, pour les étudiants assimilationnistes, ségrégationnistes ou exclusionnistes, qui sont plus ethnocentriques, les relations avec les immigrants sont plus problématiques, voire conflictuelles, car ils ont le sentiment que leur groupe est menacé par la présence des immigrants.

Les jumelages permettent la rencontre entre des personnes de différentes cultures, de différents horizons. Les deux groupes peuvent ainsi mieux se connaître. Pour Pettigrew et Tropp (2006), l'interaction entre deux groupes devrait conduire à réduire les préjugés et la discrimination. Leurs études montrent en outre que ce sont les gens du groupe majoritaire qui en tirent le plus de bénéfices.

De plus, ces jumelages permettent le développement des savoirs, des savoir-faire et des savoir-être. Ce sont les trois dimensions de la compétence interculturelle du cadre de référence de Lussier $(2005,2011)$. On entend par savoirs le contexte socioculturel et les différents modes de vie, les savoir-faire comportant les habiletés pour bien fonctionner dans la langue cible et les savoir-être comprenant trois phases : la sensibilisation culturelle, la compétence interculturelle et la compétence transculturelle. Toutes ces phases, selon Lussier $(2005,2011)$, aboutissent à une meilleure connaissance de soi, de sa culture et de sa propre identité, permettant de parvenir au respect des valeurs des autres et des autres cultures.

\section{Le soutien et le rôle de l’institution}

Les jumelages interculturels n'auraient pas pu se développer année après année sans le soutien des autorités institutionnelles. Ce soutien se traduit de différentes façons : la professeure de la Faculté des sciences de l'éducation et la directrice de l'École de langues ont, depuis les années 2000, valorisé cette activité auprès des différents enseignants et font régulièrement part des avantages des jumelages (tant chez les francophones que les nonfrancophones). Le jumelage est devenu, au fil des ans, une activité phare du programme de FLS. Plusieurs articles, communications, conférences ont eu lieu sous l'impulsion de la direction de l'École de langues. Les enseignants pratiquant l'activité sont fiers de faire part de cette rencontre entre immigrants et membres de la société d'accueil.

Par ailleurs, ce soutien s'appuie sur les politiques et les règlements en vigueur dans l'institution et repose sur la mobilisation des gestionnaires et sur le leadership partagé. Ainsi, il n'est pas inutile de rappeler qu'à UQAM, la politique sur les relations interethniques $\left(n^{\circ} 28\right)$ stipule que l'Université veut " favoriser l'intégration à la société québécoise des personnes ayant choisi de venir y vivre et contribuer activement à cette intégration » (UQAM, 2018a). De plus, les énoncés de principe de sa politique internationale ( $\left.n^{\circ} 43\right)$ mettent de l'avant "le partage de valeurs démocratiques et de principes éthiques dans le respect de la diversité des identités culturelles » (UQAM, 2018b). 
Enfin, sa la politique sur la langue française ( $n$ - 50) affirme la volonté évoque de « déployer les mesures nécessaires à l'accueil et l'intégration linguistique des non-francophones » (UQAM, 2018c).

Ces énoncés démontrent une ferme volonté institutionnelle pour développer des activités innovantes et structurantes visant de nouvelles pratiques d'enseignement et d'apprentissage. Ainsi, le jumelage n'est pas une activité " parascolaire » : c'est une activité obligatoire qui fait partie du cursus des cours et des programmes (en FLS, en formation à l'enseignement primaire et secondaire, en psychologie, en travail social, en orientation scolaire et dans tous les programmes qui participent aux jumelages). Cette volonté se traduit jusque dans l'évaluation des travaux, puisqu'à la fin du trimestre, les étudiants ont à produire une analyse décrivant leur activité de jumelage.

Outre les politiques et des règlements en vigueur à l'UQAM qui favorisent l'innovation, on peut compter sur la mobilisation des gestionnaires et sur le leadership partagé. En effet, selon Luc (2010), les personnes qui occupent des postes décisionnels ne doivent pas seulement diriger ou décider, mais elles doivent susciter la mobilisation pour un projet commun et soutenir les initiatives des autres employés. Un gestionnaire a le choix d'adopter différentes attitudes dans son mode de gestion. Celle qui s'applique ici est le leadership partagé : il « fait appel à la collaboration de tous " (Luc, 2010, p. 8). C'est ce leadership partagé qui a permis et qui permet encore la mise en place des jumelages interculturels. À titre d'exemple, lorsque le premier enseignant a voulu organiser un jumelage, le rôle du gestionnaire a été de l'écouter, de comprendre l'activité proposée et de le soutenir dans la réalisation de celle-ci. Comme le mentionne Luc (2010), pour dynamiser et faire évoluer une organisation, il faut savoir oser et « se libérer des conformismes » (p. 15). Et il a fallu oser faire des jumelages, oser penser la rencontre avec l'Autre dans le cadre d'un cours, oser reproduire cette idée et aussi oser renouveler la formule. Si la direction du département auquel appartient le programme de FLS n'avait pas appuyé, promu et encouragé le projet, le jumelage n'aurait pas pu être vécu par 15000 jumelles et jumeaux.

Luc (2010) mentionne aussi qu'un « autre moteur de libération du conformisme est le sentiment d'agir pour le bien d'une collectivité » (p. 37). Au fil du temps, les enseignants comme les gestionnaires ont adhéré et continuent d'adhérer à l'idée du vivre-ensemble et ont osé croire et continuent de croire que cette activité contribue à la cohésion sociale. Au début, certains enseignants ne se sentaient pas interpellés par une telle activité et attendaient de voir comment elle allait évoluer : il est vrai que certains d'entre eux s'étaient heurtés à des obstacles d'ordre logistique tels qu'un groupe trop petit jumelé avec un groupe trop grand, des locaux inadéquats ou des horaires incompatibles pour des cours à jumeler.

Ces obstacles n'ont pas été des freins au développement de l'activité, car la persévérance et la détermination de la direction et des enseignants qui croyaient au projet ont fait en sorte qu'il est devenu un succès, succès tel que moult étudiants en font eux-mêmes la promotion auprès des autres étudiants (qu'ils soient dans le programme de français ou dans un autre programme). Comme l'a suggéré Luc (2010), ce succès doit être partagé ouvertement, car une seule personne ne peut prendre tout le crédit de la réussite. Le développement de ce type de leadership de la part de la direction s'est également traduit par le fait qu'il a encouragé les différents enseignants à diffuser l'impact des jumelages interculturels autant pour les " accueillis " que pour les " accueillants", que ce soit par des publications, des communications ou des conférences.

De plus, pour la réalisation et le développement des activités de jumelage, une équipe s'est progressivement mobilisée : non seulement la direction du département (École de langues où l'on enseigne le français en tant que langue seconde) a tout mis en œuvre pour promouvoir le jumelage, mais deux personnes-ressources sont disponibles pour mettre en contact les enseignants qui souhaitent organiser un jumelage, pour les accompagner dans la planification et la réalisation du jumelage et répondre à leurs interrogations au cours de l'activité ou du trimestre. Ainsi, ni les enseignants ni les étudiants ne sont pas laissés à eux-mêmes, ils se sentent au contraire soutenus.

\section{L'engagement des enseignants}

Outre le soutien institutionnel, l'activité de jumelage ne peut se réaliser ni se développer sans le travail et l'engagement individuel des enseignants. Lorsqu'on présente le projet pour la première fois à un enseignant, un de ses premiers réflexes est de dire que les contenus à enseigner sont très denses et que le temps manque pour 
proposer le jumelage à ses étudiants. Organiser une telle activité, c'est sortir de l'enseignement " traditionnel » puisque l'étudiant vivra une expérience authentique dans un contexte particulier et apprendra de cet échange sans l'intervention de l'enseignant.

Au Québec, il est essentiel que les francophones, qui doivent apprendre à reconnaître et à apprécier la diversité, rencontrent des non-francophones, qui doivent pratiquer leur français en situation authentique. Pour Griggs (2009), la pédagogie par la tâche préconise que « L'enseignant fait en sorte que la tâche se substitue momentanément à luimême comme principal soutien à l'apprentissage en imposant aux élèves des buts communicatifs [...] 》 (p. 80). Pour les étudiants en éducation, en psychologie, en travail social ou en communication, le jumelage leur permettra de rencontrer et de côtoyer un immigrant.

Lors de la présentation de l'activité à un enseignant, il est important de mentionner qu'une des clés du succès consiste en la préparation du projet. Le jumelage doit s'inscrire dans les objectifs du cours afin que les étudiants perçoivent bien qu'il s'agit d'une activité intégrée au cours. Cette préparation consiste à planifier l'objectif commun, le nombre et la teneur des rencontres à l'intérieur ou à l'extérieur du cours et les critères d'évaluation à la fin du cours. Ensuite, tout au long de l'activité du jumelage, il est important de faire un retour auprès des étudiants, afin qu'ils fassent part de leurs expériences et de leurs impressions. Enfin, il est nécessaire de susciter un climat de confiance mutuel entre les enseignants, entre les enseignants et les étudiants ainsi qu'entre les étudiants, climat qui leur permettra de résoudre facilement les problèmes face aux imprévus.

\section{Les différents types de jumelage}

II n'existe pas de jumelage type, mais plutôt des types de jumelage différents, à géométrie variable. La formule va s'adapter au style des deux enseignants qui vont l'organiser, à la spécificité du cours et aux objectifs respectifs. Les rencontres, qui peuvent se faire en dyades ou en petits groupes, peuvent se dérouler pendant les heures de classe (une fois, deux fois, plusieurs fois) ou à l'extérieur des heures de classe (deux rencontres ou plus).

Par exemple, le jumelage entre des étudiants du programme de FLS et des étudiants inscrits dans un cours de psychologie se déroule actuellement pendant les heures de classe. Il consiste en une seule rencontre de trois heures : durant la première partie du cours, les étudiants de français ont comme objectif de pratiquer un extrait d'une pièce de théâtre avec les jumeaux de psychologie tandis que, durant la seconde moitié du cours, les étudiants en psychologie ont à réaliser une entrevue à faire avec leurs jumeaux ou jumelles non francophones.

Un autre exemple de jumelage propose aux étudiants des rencontres en ligne ou sur Skype afin de contrer les difficultés liées aux rencontres en présentiel. Ce jumelage en ligne entre les immigrants et les étudiants en didactique des langues secondes a permis de travailler les formules de salutation utilisées dans les courriels lors d'échanges virtuels. Conséquemment, les étudiants du programme de français ont appris les formules d'usage utilisées au Québec pendant que les étudiants de didactique des langues ont découvert les formules et les usages d’autres langues et cultures.

Enfin, un autre exemple de jumelage a lieu entre des étudiants en rédaction de textes argumentatifs et des étudiants en formation à l'enseignement du primaire et du secondaire, et il se déroule à l'extérieur des heures de cours. La prise de contact en classe permet aux étudiants d'échanger leurs coordonnées afin de planifier leurs rendez-vous pour quatre rencontres. Avant chacune d'elles, les étudiants du programme de français doivent produire un texte dans lequel ils expriment leur opinion sur un sujet controversé (par exemple, la fessée donnée aux enfants) avant de l'envoyer par courriel à leurs jumeaux ou jumelles francophones.

Ce texte sert de base de discussion au début de la rencontre. Une fois que l'étudiant francophone a corrigé les erreurs orthographiques et syntaxiques du texte produit par l'étudiant en langue, les deux jumeaux discutent du contenu et confrontent leurs points de vue. Chaque rencontre dure environ une heure et demie. Elle a lieu à l'endroit choisi par les étudiants (cafétéria, bibliothèque, restaurant, parc, domicile de l'un ou de l'autre). 
Comme nous l'avons déjà dit, à la fin du trimestre, les étudiants en formation à l'enseignement du primaire et du secondaire rédigent un rapport du jumelage présentant le parcours de leurs jumelles ou jumeaux en faisant les liens avec les différents modèles ou thématiques vus au cours (notamment parcours migratoire, ethnocentrisme, discrimination directe et indirecte, discrimination systémique, racisme, antisémitisme, islamisme, linguicisme, reconnaissance des diplômes étrangers). Pour leur part, les étudiants du cours de français produisent une synthèse qui fait état de leur expérience de jumelage. Dans les deux cas, les travaux sont sanctionnés par une évaluation incluse à la note finale du cours.

\section{Les retombées}

C'est lors de la phase de rétroaction, dernière étape du jumelage, que se mesure l'acquisition des compétences de communication interculturelle. Trimestre après trimestre, les travaux d'étudiants montrent que le jumelage interculturel développe les aspects émotifs et cognitifs des modes de communication et d'apprentissage, diminue le sentiment de menace identitaire envers les immigrants et les minorités, augmente le sentiment de sécurité linguistique et culturelle et réduit les préjugés (Bourhis, Carignan et Sioufi, 2013). Une évaluation de l'impact d'un cours de pluriethnicité incluant les jumelages interculturels a montré leur effet bénéfique auprès des étudiants québécois francophones (Bourhis, Carignan et Sioufi, 2013).

Les commentaires des étudiants sont éloquents. Pour Caroline, le jumelage a été une manière efficace d'échanger avec un immigrant et de mieux comprendre sa réalité. « II m'a permis de mieux me connaître et de mieux me définir à travers ma culture ». Pour Marco, "c'était la première fois que je faisais une activité concrète avec un francophone québécois ". Alain rappelle que Hui Min lui a fait comprendre que les immigrants veulent connaître et partager l'identité des Québécois. "Nous ne sommes pas menacés par eux, mais fortifiés par leur contact ». Pour Li Wei, arrivée au Québec depuis deux ans, c'était la première fois qu'elle rencontrait un Québécois. Chloé trouve sa jumelle russe très courageuse d'avoir fait autant d'efforts pour apprendre le français. Son désir de bien communiquer avec les enseignants de ses fils l'a beaucoup motivée. Elle gardera contact avec elle pour l'aider à améliorer son français. Michel dit qu'il va rester en contact avec son jumeau. « Le jumelage a complètement changé la vision que j'avais des immigrants ». Le jumeau de Fanny, Amir, lui a dit que rien ne vaut le contact direct pour aider à apprendre le français. Ces témoignages démontrent clairement que les jumelages contribuent à faire fondre le mur d'incompréhension et à reconnaître la richesse de la diversité ethnoculturelle dans la société québécoise.

Les jumelages interculturels sont des activités permettant un contact interculturel positif. Au lieu de s'en tenir aux discours sur l'Autre, les jumeaux et les jumelles apprennent à échanger entre eux, à se respecter, à s'apprécier et à apprendre à vivre ensemble (Carignan, Deraîche et Guillot, 2015). Les jumelages endossent le caractère réciproque de la dynamique de la communication interculturelle : il n'incombe pas qu'aux immigrants de s'adapter et de s'intégrer, c'est un travail qui concerne tout autant la population locale. Inspirés par notre approche théorique et méthodologique, des jumelages interculturels s'organisent dans des écoles secondaires en francisation et des cégeps entre anglophones et francophones ainsi que dans des organisations communautaires.

\section{Conclusion}

Depuis les années 2000, I'UQAM organise des jumelages interculturels auxquels plus de 15000 jumeaux et jumelles ont participé. II s'agit de rencontres entre des apprenants de français langue seconde et des étudiants inscrits dans des programmes disciplinaires (éducation, carriérologie, communication, psychologie, didactique des langues, entre autres). Ces rencontres, activités authentiques qui peuvent prendre différentes formes, font en sorte que des personnes de cultures différentes se rencontrent et apprennent à se connaître. Outre les assises théoriques et méthodologiques dans lesquelles s'inscrit cette activité, nous avons présenté les objectifs des jumelages interculturels et l'importance du soutien et du rôle de l'institution ainsi que celui de la mobilisation des enseignants pour la mise en place de cette activité. Celle-ci se révèle structurante sur le plan institutionnel : sept départements (provenant de trois facultés différentes) de I'UQAM y participent. Ces jumelages interculturels contribuent à l'ouverture aux autres cultures, à la diminution des préjugés et de la discrimination ainsi qu'au développement de la cohésion sociale et du vivre-ensemble. 


\section{Références bibliographiques}

Allport, G. (1954). The nature of prejudice. Cambridge : Addison-Wesley.

Bélanger, A., Sabourin, P. et Lachapelle, R. (2011). Une analyse des déterminants de la mobilité linguistique intergénérationnelle des immigrants allophones au Québec. Cahiers québécois de démographie, 40(1), 113138.

Berry, J. W. (1997). Immigration, acculturation and adaptation. Applied psychology: An International review, 46, 534.

Bourguignon, C. (2009). L'apprentissage des langues par l'action. Dans L'approche actionnelle dans l'enseignement des langues, Onze articles pour mieux comprendre et faire le point (p. 49-77). Paris : Éditions Maison des langues.

Bourhis, R., Carignan, N. et Sioufi, R. (2013). Sécurité identitaire et attitudes à l'égard de l'"Autre" chez de futurs enseignants : les impacts d'une formation interculturelle. Dans M. McAndrew et collab. (dir.), Le développement d'institutions inclusives en contexte de diversité : recherche formation, partenariat (p. 117134). Québec : Presses de l’Université du Québec.

Bourhis, R. Y., Barrette, G., El-Geledi, S. et Schmitt, R. (2009). Acculturation orientations and social relations between immigrant and host community members in California. Journal of Cross-Cultural Psychology, 40, 443-467.

Bourhis, R. Y. et El-Geledi, S. (2010). Assimilation and acculturation. Dans J. M. Levine et M. A. Hogg (dir.), Encyclopedia of Group Processes and Intergroup Relations (vol. 1, p. 30-37). Los Angeles : Sage.

Bourhis, R. Y. et Gagnon, A. (2006). Les préjugés, la discrimination et les relations intergroupes. Dans R. J. Vallerand (dir.), Les fondements de la psychologie sociale ( 2 éd., p. 532-598). Montréal : Gaëtan Morin/Chenelière Éducation.

Bourhis, R. Y. et Leyens, J.-P. (1999). Stéréotypes, discrimination et relations intergroupes ( $2^{\mathrm{e}}$ éd.). Sprimont : Madraga.

Bourhis, R. Y., Moïse, C., Perreault, S. et Senécal, S. (1997). Towards an interactive acculturation model: A social psychological approach. International Journal of Psychology, 32, 369-386.

Brown, R. (2010). Prejudice. Its social psychology ( 2 éd.). Chichester : Willey-Blackwell.

Carignan, N., Deraîche, M. et Guillot, M.-C. (2015). Jumelages interculturels : communication, inclusion et intégration. Québec: Presses de l’Université du Québec.

Croizet, J.-C. et Leyens, J.-P. (2003). Mauvaises réputations. Réalités et enjeux de la stigmatisation sociale. Paris : Armand Colin.

Griggs, P. (2009). À propos de l'articulation entre l'agir de l'usage et l'agir de l'apprentissage dans une approche actionnelle : une perspective sociocognitive. Dans L'approche actionnelle dans l'enseignement des langues, Onze articles pour mieux comprendre et faire le point (p. 56-64). Paris : Éditions Maison des langues.

James, S. et Thomas, P. (2000). John Henryism and blood pressure in Black population. A review of the evidence. African American Research Perspectives, 6, 1-10.

Johnson, D. W. et Lordan, G. (2012). Discrimination makes me sick! An examination of the discrimination-health relationship. Journal of Health Economics, 31, 99-111.

Luc, É. (2010). Le leadership partagé ( 2 éd.). Montréal : Les Presses de l’Université de Montréal.

Lussier, D. (2005). Redéfinir la compétence de communication comme une compétence de communication interculturelle. La Revue de l'AQEFLS, 25(2), 118-129.

Lussier, D. (2011). Language, thought and culture: Links to intercultural communicative competence. Canadian and International Education/Éducation canadienne et internationale, 40(2), 33-60. 
Ministère de l'Immigration, de la Diversité et de l'Inclusion (MIDI) (2014).

http://www.midi.gouv.qc.ca/fr/ministre/index.html, consulté le 25 juillet 2017.

Paluck, E. L. et Green, D. (2009). Prejudice reduction: What works? A review and assessment of research practice. Annual Review of Psychology, 60, 339-367.

Pettigrew, T. et Tropp, L. (2011). When Groups Meet: The Dynamics of Intergroup Contact. New York : Psychology Press.

Rosen, E. (2007). Le point sur le Cadre européen commun de référence pour les langues. Paris : Clé International.

Sam, D. L. et Berry J. W. (dir.) (2006). The Cambridge Handbook of Acculturation Psychology. Cambridge : Cambridge University Press.

St-Laurent, N. et El-Geledi, S. (2011). L'intégration linguistique et professionnelle des immigrants non francophones à Montréal. Rapport du Conseil supérieur de la langue française (CSLF). Montréal : Conseil supérieur de la langue française.

Tajfel, H. et Turner, J. C. (1986). The social identity theory of intergroup behaviour. Dans S. Worchel et W. G. Austin (dir.), The Psychology of Intergroup Relations (p. 7-24). Chicago : Nelson-Hall.

Université du Québec à Montréal (UQAM) (2018a). Politique no 28, Politique sur les relations interethniques. Montréal : Université du Québec à Montréal.

https://instances.uqam.ca/wp-content/uploads/sites/47/2018/05/Politique_no_28.pdf

Université du Québec à Montréal (UQAM) (2018b). Politique no 43, Politique internationale. Montréal : Université du Québec à Montréal.

https://instances.uqam.ca/wp-content/uploads/sites/47/2018/05/Politique_no_43.pdf

Université du Québec à Montréal (UQAM) (2018c). Politique no 50, Politique relative à la langue française. Montréal : Université du Québec à Montréal.

https://instances.uqam.ca/wp-content/uploads/sites/47/2018/05/Politique_no_50.pdf 